\title{
Dynamic Grid Computing
}

\author{
Edward Siedel \\ Max-Planck-Institut für Gravitationsphysik \\ Albert-Einstein-Institut \\ Haus 5 \\ Am Muehlenberg \\ 14476 Golm, Germany \\ Phone: +49-(331)-567-7210 \\ FAX: +49-(331)-567-7298 \\ eseidel@aei-potsdam.mpg.de
}

\begin{abstract}
The Grid has the potential to fundamentally change the way science and engineering are done. Aggregate power of computing resources connected by networks - of the Grid - exceeds that of any single supercomputer by many orders of magnitude. At the same time, our ability to carry out computations of the scale and level of detail required, for example, to study the Universe, or simulate a rocket engine, are severely constrained by available computing power. Hence, such applications should be one of the main driving forces behind the development of Grid computing. I will discuss some large scale applications, including simulations of colliding black holes, and show how they are driving the development of Grid computing technology. Applications are already being developed that are not only aware of their needs, but also of the resources available to them on the Grid. They will be able to adapt themselves automatically to respond to their changing needs, to spawn off tasks on other resources, and to adapt to the changing characteristics of the Grid including machine and network loads and availability. I will discuss a number of innovative scenarios for computing on the Grid enabled by such technologies, and demonstrate how close these are to being a reality.
\end{abstract}

